\title{
Optical singular and dark solitons to the nonlinear Schrodinger equation in magneto-optic waveguides with anti-cubic nonlinearity.
}

Thilagarajah Mathanaranjan ( $\nabla$ mathanaranjan@gmail.com )

University of Jaffna https://orcid.org/0000-0003-2792-4716

Hadi Rezazadeh

Amol University of Special Modern Technologies

Mehmet Senol

Nevsehir University: Nevsehir Haci Bektas Veli Universitesi

Lanre Akinyemi

Prairie View A\&M University

\section{Research Article}

Keywords: Nonlinear Schrödinger equation, Magneto-optic, Optical soliton solutions, $\left(G^{\prime}=G\right)$ - expansion method, extended tanh-function method, Modified simple equation method

Posted Date: October 5th, 2021

DOl: https://doi.org/10.21203/rs.3.rs-899309/v1

License: (9) This work is licensed under a Creative Commons Attribution 4.0 International License. Read Full License

Version of Record: A version of this preprint was published at Optical and Quantum Electronics on November 19th, 2021. See the published version at https://doi.org/10.1007/s11082-021-03383-z. 


\title{
Optical singular and dark solitons to the nonlinear Schrödinger equation in magneto-optic waveguides with anti-cubic nonlinearity
}

\author{
Thilagarajah Mathanaranjan*, Hadi Rezazadeh ${ }^{\dagger}$, Mehmet Şenol $^{\&}$, \\ Lanre Akinyemi ${ }^{\ddagger}$ \\ * Department of Mathematics and Statistics, University of Jaffna, Sri Lanka, \\ $\dagger$ Faculty of Engineering Technology, Amol University of Special Modern Technologies, \\ Amol, Iran \\ ${ }^{\&}$ Nevşehir Hacı Bektaş Veli University, Department of Mathematics, Nevşehir, Turkey \\ ${ }^{\ddagger}$ Prairie View A\&M University, Department of Mathematics, Prairie View, Texas, USA \\ E-mail: mathanaranjan@gmail.com (Thilagarajah Mathanaranjan), \\ rezazadehadi1363@gmail.com (Hadi Rezazadeh), msenol@nevsehir.edu.tr (Mehmet Şenol), \\ laakinyemi@pvamu.edu (Lanre Akinyemi).
}

\begin{abstract}
The present paper aims to investigate the coupled nonlinear Schrödinger equation (NLSE) in magneto-optic waveguides having anti-cubic (AC) law nonlinearity. The solitons secured to magneto-optic waveguides with AC law nonlinearity are extremely useful to fiber-optic transmission technology. Three constructive techniques, namely, the $\left(G^{\prime} / G\right)$-expansion method, the modified simple equation method (MSEM), and the extended tanh-function method are utilized to find the exact soliton solutions of this model. Consequently, dark, singular and combined dark-singular soliton solutions are obtained. The behaviours of soliton solutions are presented by $3 \mathrm{D}$ and $2 \mathrm{D}$ plots.
\end{abstract}

Keywords: Nonlinear Schrödinger equation; Magneto-optic; Optical soliton solutions; $\left(G^{\prime} / G\right)$ expansion method; extended tanh-function method; Modified simple equation method.

\section{Introduction}

Many nonlinear physical phenomena arise in various fields of science and engineering such as quantum mechanics, fluid dynamics, molecular biology, nuclear dynamics plasma physics, solid state physics and optical fibres. To express these complex physical phenomena in science and engineering, nonlinear partial differential equations (PDE) are widely used. Besides, it's one of the trending topics for scientists and engineers to obtain solutions for nonlinear PDE. For solving them, there are many analytical and numerical methods developed, such as the differential transform method [1], the unified method [2], the generalized exponential rational function method [3], the variational iteration method $[4,5]$, the first integral method $[6,7]$, the q-homotopy analysis method [8,9], the Riccati equation method [10], the Bernoulli collocation method [11], the residual power series method $[12,13]$, the Euler matrix method [14], the iterative shehu transform method 
[15], the Decomposition Method [16, 17], the sub-equation method [18], the $\left(G^{\prime} / G\right)$-expansion method [19, 20], the modified simple equation method [21, 22], the tanh-function method [23, 24], the solitary wave Ansatz Method [25, 26], and so on.

Recently, coupled nonlinear Schrödinger equations are studied by many scientists. The optical soliton solutions of the coupled NLSE in magneto-optic waveguides with several perturbation terms are obtained in [27]. Conservation laws for soliton propagating in birefringent optical fibers and magneto-optic waveguides are studied in [28]. A (2+1)-dimensional coupled NLSE with spatially modulated nonlinearity and transverse modulation is investigated in [29]. Bright, singular and combo optical solitons in magneto-optic waveguides with quadratic-cubic nonlinearity of coupled NLSE are obtained in [30]. Solitons in magneto-optic waveguides with parabolic law nonlinearity by Jacobi's elliptic function expansion method are developed in [31]. Solitons in magneto-optic waveguides with quadratic-cubic nonlinearity by Riccati equation expansion method are derived in [32]. Finally, solitons in magneto-optic waveguides with anti-cubic (AC) nonlinearity by Jacobi's elliptic function are extracted in [33]. In this article, our goal is to obtain the optical solitons to the NLSE in magneto-optic waveguides with AC nonlinearity, which was studied in [33], implementing the $\left(G^{\prime} / G\right)$-expansion method, the extended tanh-function method, the modified simple equation method.

The paper's structure is proposed as follows. The mathematical analysis of the proposed model is presented in Section 2. The implementations of above proposed three methods are devoted to Section 3. Finally, the last Section is reserved for the concluding remarks.

\section{Mathematical analysis of the model}

Consider the coupled NLSE in magneto-optic waveguides having AC law nonlinearity of the form $[33,34]$

$$
\begin{array}{r}
i u_{t}+a_{1} u_{x x}+\left(\frac{b_{11}}{|u|^{4}}+b_{12}|u|^{2}+b_{13}|u|^{4}+\frac{c_{11}}{|v|^{4}}+c_{12}|v|^{2}+c_{13}|v|^{4}\right) u= \\
d_{1} v+i\left[\alpha_{1} u_{x}+\lambda_{1}\left(|u|^{2} u\right)_{x}+\nu_{1}\left(|u|^{2}\right)_{x} u+\tau_{1}|u|^{2} u_{x}\right], \\
i v_{t}+a_{2} v_{x x}+\left(\frac{b_{21}}{|v|^{4}}+b_{22}|v|^{2}+b_{23}|v|^{4}+\frac{c_{21}}{|u|^{4}}+c_{22}|u|^{2}+c_{23}|u|^{4}\right) v= \\
d_{2} u+i\left[\alpha_{2} v_{x}+\lambda_{2}\left(|v|^{2} v\right)_{x}+\nu_{2}\left(|v|^{2}\right)_{x} v+\tau_{2}|v|^{2} v_{x}\right],
\end{array}
$$

where $a_{i}, b_{i 1}, b_{i 2}, b_{i 3}, c_{i 1}, c_{i 2}, c_{i 3}, d_{i}, \alpha_{i}, \lambda_{i}, \nu_{i}$, and $\tau_{i}$ are constants. Since $u(x, t)$ and $v(x, t)$ are complex function, so we assume that

$$
u(x, t)=\phi_{1}(\xi) e^{i \chi(x, t)},
$$

and

$$
v(x, t)=\phi_{2}(\xi) e^{i \chi(x, t)},
$$

where

$$
\xi=x-\nu t, \quad \chi(x, t)=-k x+\omega t
$$


and $\nu, k, \omega$ are the speed of wave, wave number and frequency of the soliton respectively. By inserting Eq. (3) into Eqs. (1) and (2), we get the real parts

$$
\begin{aligned}
& c_{11} \phi_{1}(\xi)^{4}+b_{11} \phi_{2}(\xi)^{4}+\left(b_{12}-k\left(\tau_{1}+\lambda_{1}\right)\right) \phi_{1}(\xi)^{6} \phi_{2}(\xi)^{4}+b_{13} \phi_{1}(\xi)^{8} \phi_{2}(\xi)^{4} \\
& -d_{1} \phi_{1}(\xi)^{3} \phi_{2}(\xi)^{5}-\left(\omega+k\left(k a_{1}+\alpha_{1}\right)\right) \phi_{1}(\xi)^{4} \phi_{2}(\xi)^{4}+\left(c_{12}+c_{13} \phi_{2}(\xi)^{2}\right) \phi_{1}(\xi)^{4} \phi_{2}(\xi)^{6} \\
& +a_{1} \phi_{1}(\xi)^{3} \phi_{2}(\xi)^{4} \phi_{1}^{\prime \prime}(\xi)=0 \\
& b_{21} \phi_{1}(\xi)^{4}+c_{21} \phi_{2}(\xi)^{4}+\left(b_{22}-k\left(\tau_{2}+\lambda_{2}\right)\right) \phi_{1}(\xi)^{4} \phi_{2}(\xi)^{6}+b_{23} \phi_{1}(\xi)^{4} \phi_{2}(\xi)^{8} \\
& -d_{2} \phi_{1}(\xi)^{5} \phi_{2}(\xi)^{3}-\left(\omega+k\left(k a_{2}+\alpha_{2}\right)\right) \phi_{1}(\xi)^{4} \phi_{2}(\xi)^{4}+\left(c_{22}+c_{23} \phi_{1}(\xi)^{2}\right) \phi_{1}(\xi)^{6} \phi_{2}(\xi)^{4} \\
& +a_{2} \phi_{1}(\xi)^{4} \phi_{2}(\xi)^{3} \phi_{2}^{\prime \prime}(\xi)=0,
\end{aligned}
$$

while the imaginary parts are as follows:

$$
\begin{aligned}
& \nu+2 k a_{1}+\alpha_{1}+\left(\tau_{1}+3 \lambda_{1}+2 \nu_{1}\right) \phi_{1}(\xi)^{2}=0, \\
& \nu+2 k a_{2}+\alpha_{2}+\left(\tau_{2}+3 \lambda_{2}+2 \nu_{2}\right) \phi_{2}(\xi)^{2}=0 .
\end{aligned}
$$

From Eq. (7) and Eq. (8) we get:

$$
\nu_{1}=\frac{1}{2}\left(-\tau_{1}-3 \lambda_{1}\right), \nu_{2}=\frac{1}{2}\left(-\tau_{2}-3 \lambda_{2}\right), k=\frac{-\alpha_{1}+\alpha_{2}}{2\left(a_{1}-a_{2}\right)}, \text { with } a_{1} \neq a_{2} .
$$

Now, to solve the above coupled pair of equations (5) and (6), we introduce the ansatz

$$
\phi_{2}(\xi)=K \phi_{1}(\xi),
$$

where $K(\neq 1)$ is a nonzero constant. Then, Eqs. (5) and (6) transform to

$$
\begin{aligned}
& \quad\left(c_{11}+K^{4} b_{11}\right)-K^{4}\left(\omega+K d_{1}+k\left(k a_{1}+\alpha_{1}\right)\right) \phi_{1}(\xi)^{4}+K^{4}\left(b_{12}+K^{2} c_{12}-k\left(\tau_{1}+\lambda_{1}\right)\right) \phi_{1}(\xi)^{6} \\
& \quad+K^{4}\left(b_{13}+K^{4} c_{13}\right) \phi_{1}(\xi)^{8}+K^{4} a_{1} \phi_{1}(\xi)^{3} \phi_{1}^{\prime \prime}(\xi)=0 \\
& \left(b_{21}+K^{4} c_{21}\right)-K^{3}\left(K \omega+d_{2}+k K\left(k a_{2}+\alpha_{2}\right)\right) \phi_{1}(\xi)^{4}+K^{4}\left(c_{22}+K^{2}\left(b_{22}-k\left(\tau_{2}+\lambda_{2}\right)\right)\right) \phi_{1}(\xi)^{6} \\
& +K^{4}\left(K^{4} b_{23}+c_{23}\right) \phi_{1}(\xi)^{8}+K^{3} a_{2} \phi_{1}(\xi)^{3} \phi_{1}^{\prime \prime}(\xi)=0 .
\end{aligned}
$$

Now the necessary and sufficient condition for a nontrivial solution of the function $\phi_{1}(\xi)$ satisfying both (11) and (12) is that, the coefficients of (11) and (12) satisfying the proportional ratios are as follows:

$$
\begin{aligned}
& \frac{b_{21}+K^{4} c_{21}}{c_{11}+K^{4} b_{11}}=\frac{K \omega+d_{2}+k K\left(k a_{2}+\alpha_{2}\right)}{K\left(\omega+K d_{1}+k\left(k a_{1}+\alpha_{1}\right)\right)} \\
& =\frac{c_{22}+K^{2}\left(b_{22}-k\left(\tau_{2}+\lambda_{2}\right)\right)}{b_{12}+K^{2} c_{12}-k\left(\tau_{1}+\lambda_{1}\right)}=\frac{K^{4} b_{23}+c_{23}}{b_{13}+K^{4} c_{13}}=\frac{a_{2}}{K a_{1}} .
\end{aligned}
$$

For the sake of simplicity, we set

$b_{0}=K^{3} a_{2}$,
$b_{1}=-K^{3}\left(K \omega+d_{2}+k K\left(k a_{2}+\alpha_{2}\right)\right)$, 
$b_{2}=K^{4}\left(c_{22}+K^{2}\left(b_{22}-k\left(\tau_{2}+\lambda_{2}\right)\right)\right)$,

$b_{3}=K^{4}\left(K^{4} b_{23}+c_{23}\right)$,

$b_{4}=b_{21}+K^{4} c_{21}$,

then the Eq. (12) can be written as

$$
b_{0} \phi_{1}(\xi)^{3} \phi_{1}^{\prime \prime}(\xi)+b_{1} \phi_{1}(\xi)^{4}+b_{2} \phi_{1}(\xi)^{6}+b_{3} \phi_{1}(\xi)^{8}+b_{4}=0 .
$$

Balancing $\phi_{1}^{3} \phi_{1}^{\prime \prime}$ and $\phi_{1}^{8}$ in Eq. (14), yields $N=\frac{1}{2}$. Since $N$ is not an integer, we introduce the transformation

$$
\phi_{1}(\xi)=[\psi(\xi)]^{\frac{1}{2}}
$$

where $\psi$ is a known function of $\xi$. Inserting Eq. (15) into Eq. (14), we obtain the new equation

$$
2 b_{0} \psi(\xi) \psi^{\prime \prime}(\xi)-b_{0} \psi^{\prime}(\xi)^{2}+4 b_{1} \psi(\xi)^{2}+4 b_{2} \psi(\xi)^{3}+4 b_{3} \psi(\xi)^{4}+4 b_{4}=0
$$

Now, our task is to solve Eq.(16) by above mentioned three different analytical methods.

\section{Solitons to the NLSE in magneto-optic waveguides with anti-cubic nonlinearity}

In this section, we apply the proposed three methods to find the soliton solutions of NLSE in magneto-optic waveguides having AC nonlinearity.

\subsection{Applying the $\left(G^{\prime} / G\right)$-expansion method}

From the homogeneous balance of $\psi(\xi) \psi^{\prime \prime}(\xi)$ and $\psi(\xi)^{4}$ in Eq. (16), we find $N=1$. According to the proposed method [19, 20], the solution of Eq.(16) is given as:

$$
\psi(\xi)=\alpha_{0}+\alpha_{1}\left(G^{\prime} / G\right)
$$

where the constants $\alpha_{0}$, and $\alpha_{1}$ are to be determined later and $G(\xi)$ satisfies the following ordinary differential equation:

$$
G^{\prime \prime}(\xi)+\lambda G^{\prime}(\xi)+\mu G(\xi)=0
$$

here $\lambda$ and $\mu$ are constants. Substituting Eq. (17) together with Eq. (18) into Eq. (16), and making the coefficients of $\left(G^{\prime} / G\right)^{N}(N=0,1,2,3,4)$ equal to zero, yield the system of algebraic equations as follows:

$\left[G^{\prime} / G\right]^{0}: \quad 4 b_{4}+4 \alpha_{0}^{2}\left(b_{1}+\alpha_{0}\left(b_{2}+b_{3} \alpha_{0}\right)\right)+2 \lambda \mu b_{0} \alpha_{0} \alpha_{1}-\mu^{2} b_{0} \alpha_{1}^{2}=0$,

$\left[G^{\prime} / G\right]^{1}: 2 \alpha_{0}\left(\left(\lambda^{2}+2 \mu\right) b_{0}+4 b_{1}+6 b_{2} \alpha_{0}+8 b_{3} \alpha_{0}^{2}\right) \alpha_{1}=0$

$\left[G^{\prime} / G\right]^{2}: \quad \alpha_{1}\left(4\left(b_{1}+3 \alpha_{0}\left(b_{2}+2 b_{3} \alpha_{0}\right)\right) \alpha_{1}+b_{0}\left(6 \lambda \alpha_{0}+\left(\lambda^{2}+2 \mu\right) \alpha_{1}\right)\right)=0$

$\left[G^{\prime} / G\right]^{3}: \quad 4 \alpha_{1}\left(\left(b_{2}+4 b_{3} \alpha_{0}\right) \alpha_{1}^{2}+b_{0}\left(\alpha_{0}+\lambda \alpha_{1}\right)\right)=0$,

$\left[G^{\prime} / G\right]^{4}: \quad 3 b_{0} \alpha_{1}^{2}+4 b_{3} \alpha_{1}^{4}=0$. 
The following outcome is found by solving the above equations:

$$
\alpha_{0}=0, \alpha_{1}= \pm \frac{1}{2} \sqrt{-\frac{3 b_{0}}{b_{3}}}, \lambda=\mp \frac{b_{2}}{2} \sqrt{-\frac{3}{b_{0} b_{3}}}, \mu=\frac{3 b_{2}^{2}-16 b_{1} b_{3}}{8 b_{0} b_{3}}, b_{4}=-\frac{3\left(3 b_{2}^{2}-16 b_{1} b_{3}\right)^{2}}{1024 b_{3}^{3}},
$$

Provided that $b_{0} b_{3}<0$. By inserting Eq.(20) into Eq.(17), we obtain

$$
\psi(\xi)= \pm \frac{1}{2} \sqrt{-\frac{3 b_{0}}{b_{3}}}\left(G^{\prime} / G\right) .
$$

Inserting general solutions of Eq. (18) into Eqs. (21), we obtain three types of travelling wave solutions to the coupled pair of Eq. (1) and Eq. (2) as:

Type 1. When $\lambda^{2}-4 \mu=\frac{-9 b_{2}^{2}+32 b_{1} b_{3}}{4 b_{0} b_{3}}>0$, we obtain the hyperbolic function solutions of Eq. (1) and Eq. (2) as:

$$
\begin{aligned}
u(x, t)= & -\frac{3 b_{2}}{8 b_{3}} \pm \frac{\sqrt{3} \sqrt{9 b_{2}^{2}-32 b_{1} b_{3}}}{8 b_{3}}\left(\frac{c_{1} \sinh \left[\frac{1}{4} \xi \sqrt{\frac{-9 b_{2}^{2}+32 b_{1} b_{3}}{b_{0} b_{3}}}\right]+c_{2} \cosh \left[\frac{1}{4} \xi \sqrt{\frac{-9 b_{2}^{2}+32 b_{1} b_{3}}{b_{0} b_{3}}}\right]}{c_{1} \cosh \left[\frac{1}{4} \xi \sqrt{\frac{-9 b_{2}^{2}+32 b_{1} b_{3}}{b_{0} b_{3}}}\right]+c_{2} \sinh \left[\frac{1}{4} \xi \sqrt{\frac{-9 b_{2}^{2}+32 b_{1} b_{3}}{b_{0} b_{3}}}\right]}\right)^{1 / 2} \\
& \times \exp (i(-k x+\omega t))
\end{aligned}
$$

and $v(x, t)=K u(x, t)$.

In particular, if we choose " $c_{1} \neq 0, c_{2}=0$ " in $(22)$, then the dark soliton solution is revealed as:

$$
u(x, t)=\left(-\frac{3 b_{2}}{8 b_{3}} \pm \frac{\sqrt{3} \sqrt{9 b_{2}^{2}-32 b_{1} b_{3}}}{8 b_{3}} \tanh \left[\frac{1}{4} \xi \sqrt{\frac{-9 b_{2}^{2}+32 b_{1} b_{3}}{b_{0} b_{3}}}\right]\right)^{1 / 2} \exp (i(-k x+\omega t)) .
$$

If we choose " $c_{1}=0, c_{2} \neq 0$ " in (22), then the singular soliton solution falls out:

$$
u(x, t)=\left(-\frac{3 b_{2}}{8 b_{3}} \pm \frac{\sqrt{3} \sqrt{9 b_{2}^{2}-32 b_{1} b_{3}}}{8 b_{3}} \operatorname{coth}\left[\frac{1}{4} \xi \sqrt{\frac{-9 b_{2}^{2}+32 b_{1} b_{3}}{b_{0} b_{3}}}\right]\right)^{1 / 2} \exp (i(-k x+\omega t)) .
$$

The behaviours of soliton solutions of Eq. (23) and (24) are presented in Fig. 1 and Fig. 2 respectively.

Type 2. When $\lambda^{2}-4 \mu=\frac{-9 b_{2}^{2}+32 b_{1} b_{3}}{4 b_{0} b_{3}}<0$, we obtain the trigonometric function solutions of Eq. (1) and Eq. (2) as follows:

$$
\begin{aligned}
u(x, t)= & \left.=\frac{3 b_{2}}{8 b_{3}} \pm \frac{\sqrt{3} \sqrt{-9 b_{2}^{2}+32 b_{1} b_{3}}}{8 b_{3}}\left(\frac{c_{1} \sin \left[\frac{1}{4} \xi \sqrt{\frac{9 b_{2}^{2}-32 b_{1} b_{3}}{b_{0} b_{3}}}\right]+c_{2} \cos \left[\frac{1}{4} \xi \sqrt{\frac{9 b_{2}^{2}-32 b_{1} b_{3}}{b_{0} b_{3}}}\right]}{c_{1} \cos \left[\frac{1}{4} \xi \sqrt{\frac{9 b_{2}^{2}-32 b_{1} b_{3}}{b_{0} b_{3}}}\right]+c_{2} \sin \left[\frac{1}{4} \xi \sqrt{\frac{9 b_{2}^{2}-32 b_{1} b_{3}}{b_{0} b_{3}}}\right]}\right)\right)^{1 / 2} \\
& \quad \times \exp (i(-k x+\omega t)),
\end{aligned}
$$



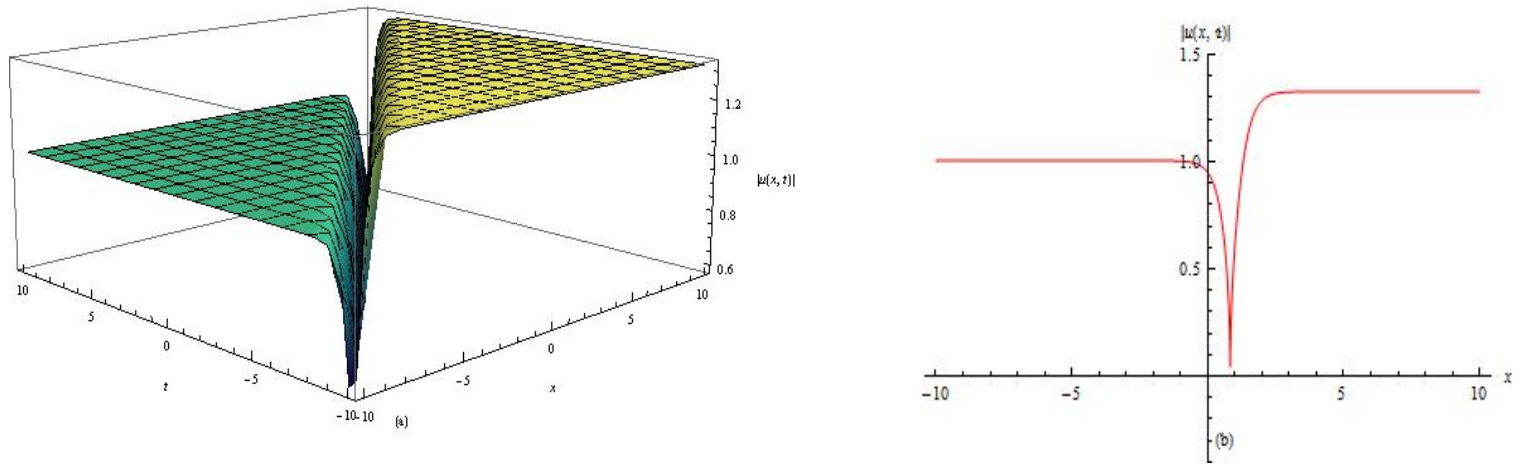

Figure 1: (a) 3D graph of Eq. (23) with $K=\omega=k=a_{2}=\alpha_{2}=\theta_{2}=\lambda_{2}=b_{22}=b_{23}=1, b_{21}=c_{22}=$ $2, c_{21}=-1, c_{23}=-2, d_{2}=-4$ (b) Corresponding $2 \mathrm{D}$ graph for $t=1$.
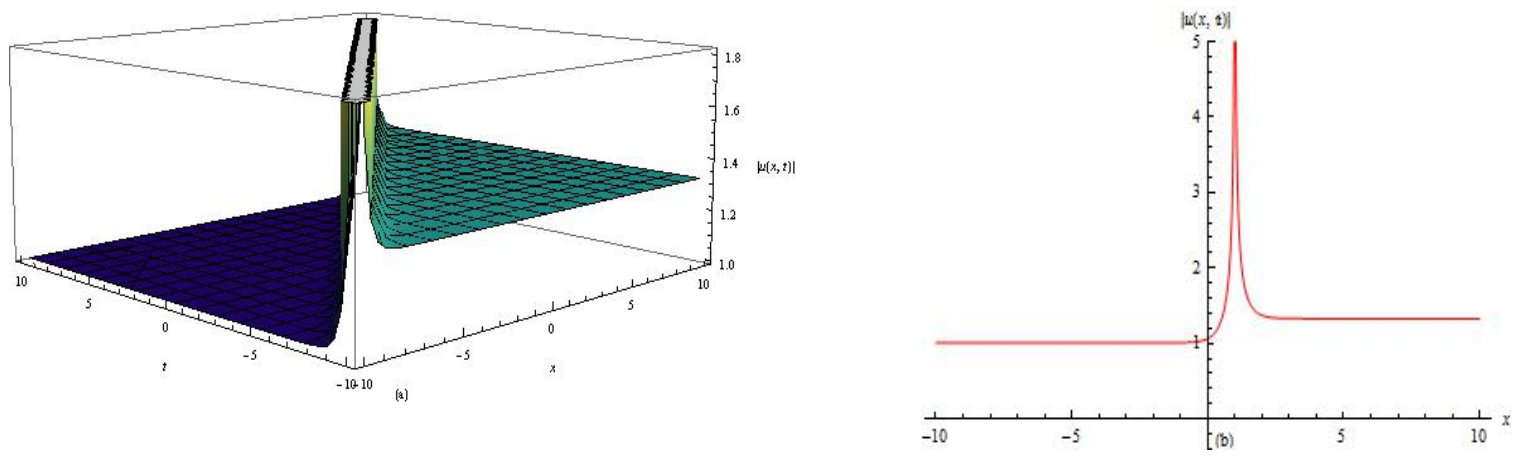

Figure 2: (a) 3D graph of Eq. (24) with $K=\omega=k=a_{2}=\alpha_{2}=\theta_{2}=\lambda_{2}=b_{22}=b_{23}=1, b_{21}=c_{22}=$ $2, c_{21}=-1, c_{23}=-2, d_{2}=-4$ (b) Corresponding $2 \mathrm{D}$ graph for $t=1$.

and $v(x, t)=K u(x, t)$.

In particular, if we choose " $c_{1} \neq 0, c_{2}=0$ " in (22), then the periodic solitary wave solutions is reveled as:

$$
u(x, t)=\left(-\frac{3 b_{2}}{8 b_{3}} \pm \frac{\sqrt{3} \sqrt{-9 b_{2}^{2}+32 b_{1} b_{3}}}{8 b_{3}} \tan \left[\frac{1}{4} \xi \sqrt{\frac{9 b_{2}^{2}-32 b_{1} b_{3}}{b_{0} b_{3}}}\right]\right)^{1 / 2} \exp (i(-k x+\omega t)) .
$$

If we choose " $c_{1}=0, c_{2} \neq 0$ " in $(22)$, then the periodic solitary wave solutions falls out:

$$
u(x, t)=\left(-\frac{3 b_{2}}{8 b_{3}} \pm \frac{\sqrt{3} \sqrt{-9 b_{2}^{2}+32 b_{1} b_{3}}}{8 b_{3}} \cot \left[\frac{1}{4} \xi \sqrt{\frac{9 b_{2}^{2}-32 b_{1} b_{3}}{b_{0} b_{3}}}\right]\right)^{1 / 2} \exp (i(-k x+\omega t))
$$

The behaviours of soliton solutions of Eq. (26) and (27) are presented in Fig. 3 and Fig. 4 respectively. 

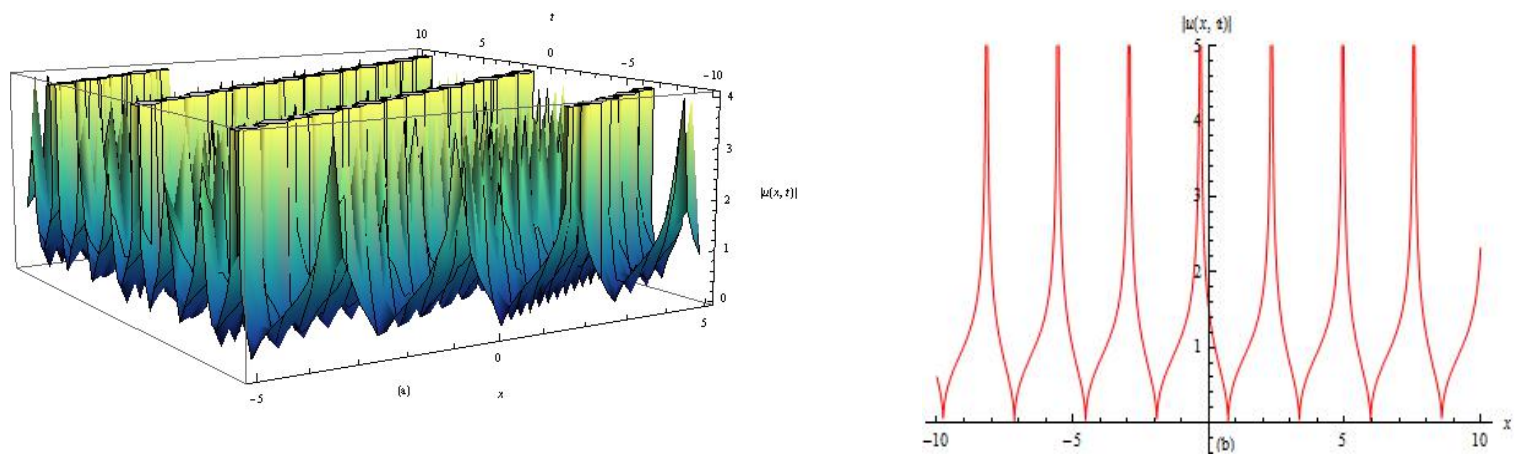

Figure 3: (a) 3D graph of Eq. (26) with $K=\omega=k=\theta_{2}=\lambda_{2}=b_{22}=1, a_{2}=b_{23}=c_{21}=-1, \alpha_{2}=$ $3, b_{21}=c_{22}=c_{23}=2, d_{2}=-4(\mathrm{~b})$ Corresponding $2 \mathrm{D}$ graph for $t=1$.
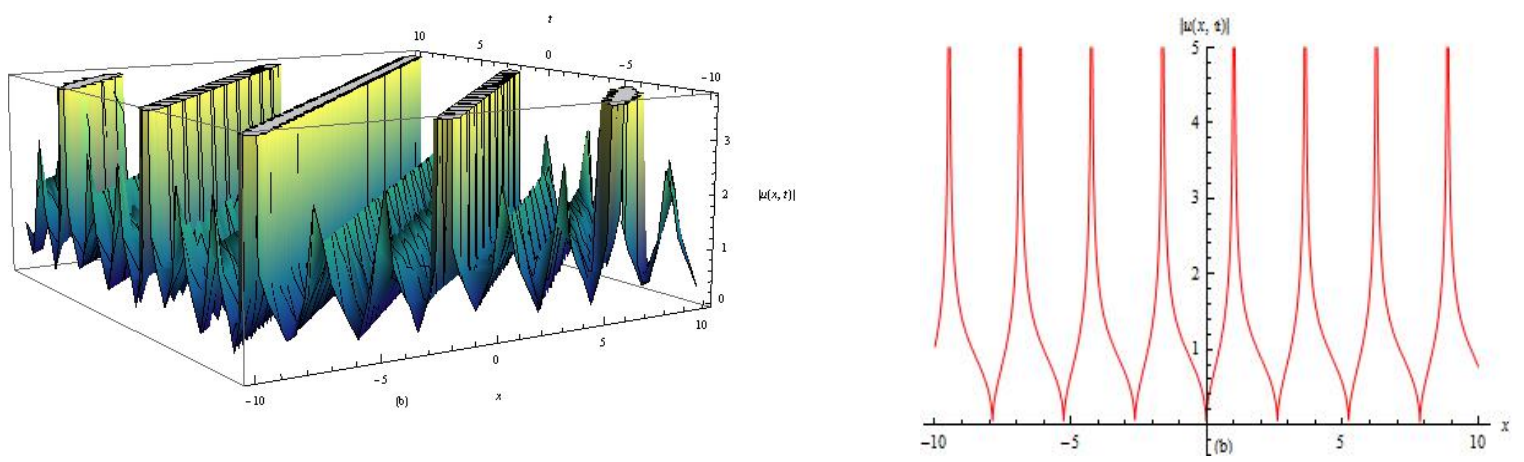

Figure 4: (a) 3D graph of Eq. (27) with $K=\omega=k=\theta_{2}=\lambda_{2}=b_{22}=1, a_{2}=b_{23}=c_{21}=-1, \alpha_{2}=$ $3, b_{21}=c_{22}=c_{23}=2, d_{2}=-4$ (b) Corresponding 2D graph for $t=1$.

Type 3. When $\lambda^{2}-4 \mu=\frac{-9 b_{2}^{2}+32 b_{1} b_{3}}{4 b_{0} b_{3}}=0$, we obtain the rational function solutions as follows:

$$
u(x, t)=\left(-\frac{3 b_{2}}{8 b_{3}} \pm \frac{1}{2} \sqrt{-\frac{3 b_{0}}{b_{3}}} \frac{c_{2}}{c_{1}+c_{2} \xi}\right)^{1 / 2} \exp (i(-k x+\omega t)) .
$$

and $v(x, t)=K u(x, t)$, where $c_{1}$ and $c_{2}$ are constants.

\subsection{Applying the modified simple equation method}

Considering the homogeneous balance among $\psi(\xi) \psi^{\prime \prime}(\xi)$ and $\psi(\xi)^{4}$ in Eq.(16), one get $N=1$. Based on the MSEM [21, 22], we suppose that:

$$
\psi(\xi)=A_{0}+A_{1}\left[\frac{\Psi^{\prime}(\xi)}{\Psi(\xi)}\right]
$$

where $A_{0}$ and $A_{1} \neq 0$ are constants to be determined later. Inserting (29) into (16), and then setting the coefficients $\Psi(\xi)^{-j}(j=0,1,2,3,4)$ equal to zero, we find a set of algebraic equations 
as follows:

$$
\begin{aligned}
\Psi(\xi)^{0}: & 4\left(b_{4}+\alpha_{0}^{2}\left(b_{1}+\alpha_{0}\left(b_{2}+b_{3} \alpha_{0}\right)\right)\right)=0, \\
\Psi(\xi)^{-1}: & 22 \alpha_{0} \alpha_{1}\left(2\left(2 b_{1}+\alpha_{0}\left(3 b_{2}+4 b_{3} \alpha_{0}\right)\right) \Psi^{\prime}(\xi)+b_{0} \Psi^{(3)}(\xi)\right)=0, \\
\Psi(\xi)^{-2}: & \alpha_{1}\left(4\left(b_{1}+3 \alpha_{0}\left(b_{2}+2 b_{3} \alpha_{0}\right)\right) \alpha_{1} \Psi^{\prime}(\xi)^{2}-6 b_{0} \alpha_{0} \Psi^{\prime}(\xi) \Psi^{\prime \prime}(\xi)-b_{0} \alpha_{1} \Psi^{\prime \prime}(\xi)^{2}\right. \\
& \left.+2 b_{0} \alpha_{1} \Psi^{\prime}(\xi) \Psi^{(3)}(\xi)\right)=0, \\
\Psi(\xi)^{-3}: & 4 \alpha_{1} \Psi^{\prime}(\xi)^{2}\left(\left(b_{0} \alpha_{0}+\left(b_{2}+4 b_{3} \alpha_{0}\right) \alpha_{1}^{2}\right) \Psi^{\prime}(\xi)-b_{0} \alpha_{1} \Psi^{\prime \prime}(\xi)\right)=0, \\
\Psi(\xi)^{-4}: & \alpha_{1}^{2}\left(3 b_{0}+4 b_{3} \alpha_{1}^{2}\right) \Psi^{\prime}(\xi)^{4}=0 .
\end{aligned}
$$

Solving above algebraic equations (30), we obtain,

$$
\alpha_{1}= \pm \frac{1}{2} \sqrt{-\frac{3 b_{0}}{b_{3}}}, \alpha_{0}=\frac{-3 b_{2} \mp \sqrt{3} \sqrt{9 b_{2}^{2}-32 b_{1} b_{3}}}{8 b_{3}}, b_{4}=-\frac{3\left(3 b_{2}^{2}-16 b_{1} b_{3}\right)^{2}}{1024 b_{3}^{3}}
$$

and

$$
9 b_{2}^{2} \Psi^{\prime}(\xi)+4 b_{3}\left(-8 b_{1} \Psi^{\prime}(\xi)+b_{0} \Psi^{(3)}(\xi)\right)=0,
$$

provided that $b_{0} b_{3}<0$ and $\left(9 b_{2}^{2}-32 b_{1} b_{3}\right)>0$. Consequently, the equation (32) reduces to

$$
\Psi(\xi)=c_{3}+\frac{2 e^{-\frac{\xi}{2} \sqrt{\frac{-9 b_{2}^{2}+32 b_{1} b_{3}}{b_{0} b_{3}}}}\left[-c_{1}+c_{2} e^{\left.\xi \sqrt{\frac{-9 b_{2}^{2}+32 b_{1} b_{3}}{b_{0} b_{3}}}\right] \sqrt{b_{0} b_{3}}}\right.}{\sqrt{-9 b_{2}^{2}+32 b_{1} b_{3}}},
$$

where $c_{1}, c_{2}$ and $c_{3}$ are constants. Substituting (33) into (3) with (15), we find the exact solution to coupled pair of Eq.(1) and Eq.(2) as follows:

$$
\begin{aligned}
& u(x, t)=\left(-\frac{3 b_{2}}{8 b_{3}} \pm \frac{\sqrt{3} \sqrt{9 b_{2}^{2}-32 b_{1} b_{3}}}{8 b_{3}} \mp \frac{\frac{\sqrt{3} \sqrt{9 b_{2}^{2}-32 b_{1} b_{3}}}{4 b_{3}}\left(c_{1}+c_{2} e^{\xi \sqrt{\frac{-9 b_{2}^{2}+32 b_{1} b_{3}}{b_{0} b_{3}}}}\right)}{-c_{1}+c_{2} e^{\xi \sqrt{\frac{-9 b_{2}^{2}+32 b_{1} b_{3}}{b_{0} b_{3}}}}+\sqrt{\frac{-9 b_{2}^{2}+32 b_{1} b_{3}}{4 b_{0} b_{3}}} c_{3} e^{\frac{\xi}{2} \sqrt{\frac{-9 b_{2}^{2}+32 b_{1} b_{3}}{b_{0} b_{3}}}}}\right)^{1 / 2} \\
& \times \exp (i(-k x+\omega t)),
\end{aligned}
$$

and $v(x, t)=K u(x, t)$.

As a particular selection, if we take $c_{1}=0, \frac{c_{3}}{c_{2}}=\sqrt{\frac{4 b_{0} b_{3}}{-9 b_{2}^{2}+32 b_{1} b_{3}}}$ in (34), the dark soliton solution can be obtained as:

$$
u(x, t)=\left(-\frac{3 b_{2}}{8 b_{3}} \pm \frac{\sqrt{3} \sqrt{9 b_{2}^{2}-32 b_{1} b_{3}}}{8 b_{3}} \tanh \left[\frac{1}{4} \xi \sqrt{\frac{-9 b_{2}^{2}+32 b_{1} b_{3}}{b_{0} b_{3}}}\right]\right)^{1 / 2} \exp (i(-k x+\omega t)),
$$


while, if we set $c_{1}=0, \frac{c_{3}}{c_{2}}=-\sqrt{\frac{4 b_{0} b_{3}}{-9 b_{2}^{2}+32 b_{1} b_{3}}}$ in $(34)$, singular soliton solution can be obtained as:

$$
u(x, t)=\left(-\frac{3 b_{2}}{8 b_{3}} \pm \frac{\sqrt{3} \sqrt{9 b_{2}^{2}-32 b_{1} b_{3}}}{8 b_{3}} \operatorname{coth}\left[\frac{1}{4} \xi \sqrt{\frac{-9 b_{2}^{2}+32 b_{1} b_{3}}{b_{0} b_{3}}}\right]\right)^{1 / 2} \exp (i(-k x+\omega t)) .
$$

It should be noted that the solitary wave solutions (35) and (36) are similar to the previous solutions (23) and (24) respectively.

\subsection{Applying the extended tanh-function method}

According to the proposed method [23, 24], we assume that

$$
Y=\tanh (\mu \xi)
$$

which gives to the change of variables

$$
\begin{gathered}
\frac{d \psi}{d \xi}=\mu\left(1-Y^{2}\right) \frac{d \psi}{d Y} \\
\frac{d^{2} \psi}{d \xi^{2}}=-2 \mu^{2} Y\left(1-Y^{2}\right) \frac{d \psi}{d Y}+\mu^{2}\left(1-Y^{2}\right)^{2} \frac{d^{2} \psi}{d Y^{2}}
\end{gathered}
$$

The solution of above equation is considered as

$$
\psi(\xi)=\sum_{k=-N}^{N} a_{k} Y^{k}
$$

where $N$ is balancing number. Considering the homogeneous balance among $\psi(\xi) \psi^{\prime \prime}(\xi)$ and $\psi(\xi)^{4}$ in Eq. (16), provides $N=1$. Then, from Eq. (40)

$$
\psi(\xi)=\alpha_{0}+\alpha_{1} Y+\frac{\alpha_{-1}}{Y} .
$$

Substituting (41) into (16), and making all the coefficients of powers $Y$ equal to zero, we get a set of equations as follows:

$$
\begin{aligned}
Y^{-4}: & 3 \mu^{2} b_{0} \alpha_{-1}^{2}+4 b_{3} \alpha_{-1}^{4}=0, \\
Y^{-3}: & 4\left(b_{2} \alpha_{-1}^{3}+\alpha_{-1}\left(\mu^{2} b_{0}+4 b_{3} \alpha_{-1}^{2}\right) \alpha_{0}\right)=0, \\
Y^{-2}: & 2 \alpha_{-1}\left(-\mu^{2} b_{0}\left(\alpha_{-1}-3 \alpha_{1}\right)+2 \alpha_{-1}\left(b_{1}+3 \alpha_{0}\left(b_{2}+2 b_{3} \alpha_{0}\right)+4 b_{3} \alpha_{-1} \alpha_{1}\right)\right)=0, \\
Y^{-1}: & 4 \alpha_{-1}\left(\alpha_{0}\left(-\mu^{2} b_{0}+2 b_{1}+\alpha_{0}\left(3 b_{2}+4 b_{3} \alpha_{0}\right)\right)+3 \alpha_{-1}\left(b_{2}+4 b_{3} \alpha_{0}\right) \alpha_{1}\right)=0, \\
Y^{0}: & 4 b_{4}+4 \alpha_{0}^{2}\left(b_{1}+\alpha_{0}\left(b_{2}+b_{3} \alpha_{0}\right)\right)+8 \alpha_{-1} \alpha_{1}\left(b_{1}+3 \alpha_{0}\left(b_{2}+2 b_{3} \alpha_{0}\right)+3 b_{3} \alpha_{-1} \alpha_{1}\right) \\
& -\mu^{2} b_{0}\left(\alpha_{-1}^{2}+12 \alpha_{-1} \alpha_{1}+\alpha_{1}^{2}\right)=0, \\
Y^{1}: & 4 \alpha_{1}\left(\alpha_{0}\left(-\mu^{2} b_{0}+2 b_{1}+\alpha_{0}\left(3 b_{2}+4 b_{3} \alpha_{0}\right)\right)+3 \alpha_{-1}\left(b_{2}+4 b_{3} \alpha_{0}\right) \alpha_{1}\right)=0, \\
Y^{2}: & 2 \alpha_{1}\left(\mu^{2} b_{0}\left(3 \alpha_{-1}-\alpha_{1}\right)+2 \alpha_{1}\left(b_{1}+3 \alpha_{0}\left(b_{2}+2 b_{3} \alpha_{0}\right)+4 b_{3} \alpha_{-1} \alpha_{1}\right)\right)=0, \\
Y^{3}: & 4\left(\mu^{2} b_{0} \alpha_{0} \alpha_{1}+\left(b_{2}+4 b_{3} \alpha_{0}\right) \alpha_{1}^{3}\right)=0, \\
Y^{4}: & 3 \mu^{2} b_{0} \alpha_{1}^{2}+4 b_{3} \alpha_{1}^{4}=0 .
\end{aligned}
$$

Solving the above system, we find the following three sets of solutions: 
1. The first set:

$$
\begin{aligned}
\alpha_{0} & =-\frac{3 b_{2}}{8 b_{3}}, \quad \alpha_{-1}=0, & \alpha_{1} & = \pm \frac{\sqrt{3} \sqrt{9 b_{2}^{2}-32 b_{1} b_{3}}}{8 b_{3}} \\
\mu & = \pm \frac{1}{4} \sqrt{\frac{-9 b_{2}^{2}+32 b_{1} b_{3}}{b_{0} b_{3}}}, & b_{4} & =-\frac{3\left(3 b_{2}^{2}-16 b_{1} b_{3}\right)^{2}}{1024 b_{3}^{3}}
\end{aligned}
$$

2. The second set:

$$
\begin{aligned}
\alpha_{0} & =-\frac{3 b_{2}}{8 b_{3}}, \quad \alpha_{-1}= \pm \frac{\sqrt{3} \sqrt{9 b_{2}^{2}-32 b_{1} b_{3}}}{8 b_{3}}, \quad \alpha_{1}=0 \\
\mu & = \pm \frac{1}{4} \sqrt{\frac{-9 b_{2}^{2}+32 b_{1} b_{3}}{b_{0} b_{3}}}, \quad b_{4}=-\frac{3\left(3 b_{2}^{2}-16 b_{1} b_{3}\right)^{2}}{1024 b_{3}^{3}}
\end{aligned}
$$

3. The third set:

$$
\begin{aligned}
& \alpha_{0}=-\frac{3 b_{2}}{8 b_{3}}, \quad \alpha_{1}=\alpha_{-1}= \pm \frac{\sqrt{3} \sqrt{9 b_{2}^{2}-32 b_{1} b_{3}}}{16 b_{3}} \\
& \mu= \pm \frac{1}{8} \sqrt{\frac{-9 b_{2}^{2}+32 b_{1} b_{3}}{b_{0} b_{3}}}, \quad b_{4}=-\frac{3\left(3 b_{2}^{2}-16 b_{1} b_{3}\right)^{2}}{1024 b_{3}^{3}} .
\end{aligned}
$$

Provided that $b_{0} b_{3}<0$ and $\left(9 b_{2}^{2}-32 b_{1} b_{3}\right)>0$. From Eq. (43) - (45), the dark, singular and combined dark-singular soliton solutions to the coupled pair of Eq. (1) and (2) read as follows:

$$
\begin{aligned}
& u(x, t)=\left(-\frac{3 b_{2}}{8 b_{3}} \pm \frac{\sqrt{3} \sqrt{9 b_{2}^{2}-32 b_{1} b_{3}}}{8 b_{3}} \tanh \left[\frac{1}{4} \xi \sqrt{\frac{-9 b_{2}^{2}+32 b_{1} b_{3}}{b_{0} b_{3}}}\right]\right)^{1 / 2} \exp (i(-k x+\omega t)), \\
& u(x, t)=\left(-\frac{3 b_{2}}{8 b_{3}} \pm \frac{\sqrt{3} \sqrt{9 b_{2}^{2}-32 b_{1} b_{3}}}{8 b_{3}} \operatorname{coth}\left[\frac{1}{4} \xi \sqrt{\frac{-9 b_{2}^{2}+32 b_{1} b_{3}}{b_{0} b_{3}}}\right]\right)^{1 / 2} \exp (i(-k x+\omega t)),
\end{aligned}
$$

$$
\begin{aligned}
u(x, t)=\left(-\frac{3 b_{2}}{8 b_{3}} \pm \frac{\sqrt{3} \sqrt{9 b_{2}^{2}-32 b_{1} b_{3}}}{16 b_{3}}\left(\tanh \left[\frac{1}{8} \xi \sqrt{\frac{-9 b_{2}^{2}+32 b_{1} b_{3}}{b_{0} b_{3}}}\right]+\operatorname{coth}\left[\frac{1}{8} \xi \sqrt{\frac{-9 b_{2}^{2}+32 b_{1} b_{3}}{b_{0} b_{3}}}\right]\right)\right)^{1 / 2} \\
\quad \times \exp (i(-k x+\omega t))
\end{aligned}
$$

and $v(x, t)=K u(x, t)$.

Note that the first two solutions are equivalent to the previous solutions (23) and (24) respectively. The behaviour of soliton solution of Eq. (48) is shown in Fig. 5. 

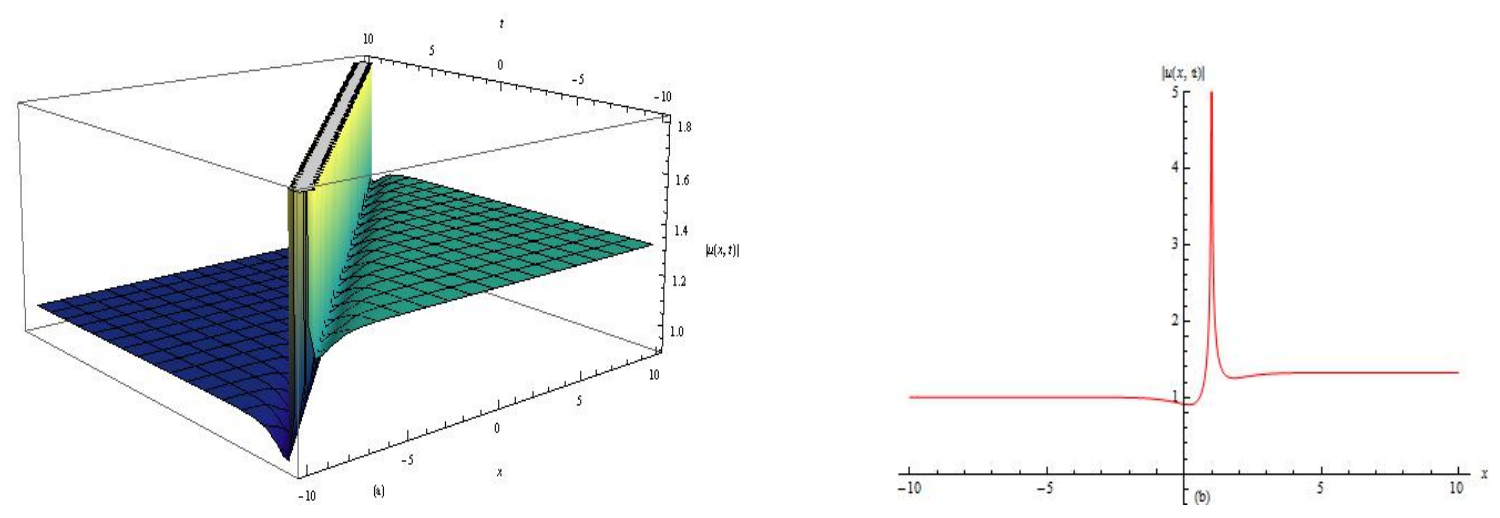

Figure 5: (a) 3D graph of Eq. (48) with $K=\omega=k=a_{2}=\alpha_{2}=\theta_{2}=\lambda_{2}=b_{22}=b_{23}=1, b_{21}=c_{22}=$ $2, c_{21}=-1, c_{23}=-2, d_{2}=-4$ (b) Corresponding 2D graph for $t=1$.

\section{Conclusions}

This study investigates the optical solitons solution of the nonlinear Schrödinger equation in magneto-optic waveguides with AC nonlinearity. The solitons secured to magneto-optic waveguides with AC law nonlinearity will be extremely advantageous in fiber-optic transmission technology. Therefore, based on three effective methods, namely the $\left(G^{\prime} / G\right)$-expansion method, the modified simple equation method and the extended tanh-function method, we have successfully obtained the dark, singular and combined dark-singular soliton solutions of the above model. The obtained solutions are illustrated by 3D and 2D graphs to express the dynamical behaviour of solutions.

\section{References}

[1] Akinyemi, L.A. (2020). fractional analysis of Noyes-Field model for the nonlinear Belousov-Zhabotinsky reaction. Comp. Appl. Math., 39, 1-34. https://doi.org/10.1007/s40314020-01212-9

[2] Osman, M. S., Korkmaz, A., Rezazadeh, H., Mirzazadeh, M., Eslami, M., \& Zhou, Q. (2018). The unified method for conformable time fractional Schro" dinger equation with perturbation terms. Chinese Journal of Physics, 56(5), 2500-2506.

[3] Ghanbari, B., Osman, M. S., \& Baleanu, D. (2019). Generalized exponential rational function method for extended Zakharov-Kuzetsov equation with conformable derivative. Modern Physics Letters A, 34(20), 1950155.

[4] Das, S. (2009). Analytical solution of a fractional diffusion equation by variational iteration method. Comput. Math. Appl., 57, 483-487.

[5] He, J.H. (1998). Approximate analytical solution for seepage flow with fractional derivatives in porous media. Comput. Meth. Appl. Mech. Eng., 167, 57-68.

[6] Eslami, M., \& Rezazadeh, H. (2016). The first integral method for Wu-Zhang system with conformable time-fractional derivative. Calcolo, 53(3), 475-485. 
[7] Mathanaranjan. T. (2021). Exact and explicit traveling wave solutions to the generalized Gardner and BBMB equations with dual high-order nonlinear terms. To be published in: Partial Differential Equations in Applied Mathematics.

[8] Akinyemi, L. (2019). q-Homotopy analysis method for solving the seventh-order timefractional Lax's Korteweg-de Vries and Sawada-Kotera equations. Comp. Appl. Math., 38(4), $1-22$.

[9] Akinyemi, L., Iyiola, O.S., Akpan, U. (2020). Iterative methods for solving fourth- and sixth order time-fractional Cahn-Hillard equation. Math. Meth. Appl. Sci., 43(7), 4050-4074. https://doi.org/10.1002/mma.6173

[10] Rezazadeh, H., Korkmaz, A., Eslami, M., Vahidi, J., \& Asghari, R. (2018). Traveling wave solution of conformable fractional generalized reaction Duffing model by generalized projective Riccati equation method. Optical and Quantum Electronics, 50(3), 150.

[11] Adel, W., \& Sabir, Z. (2020). Solving a new design of nonlinear second-order Lane-Emden pantograph delay differential model via Bernoulli collocation method. The European Physical Journal Plus, 135(6), 427.

[12] Senol, M., Iyiola, O.S., Daei Kasmaei, H., Akinyemi, L. (2019). Efficient analytical techniques for solving time-fractional nonlinear coupled Jaulent-Miodek system with energy-dependent Schrödinger potential. Adv. Differ. Equ., 2019, 1-21.

[13] Senol, M. (2020). Analytical and approximate solutions of $(2+1)$-dimensional time-fractional Burgers-Kadomtsev-Petviashvili equation. Commun. Theor. Phys., 72(5), 1-11.

[14] El-Gamel, M., \& Adel, W. (2018). Numerical investigation of the solution of higher-order boundary value problems via Euler matrix method. SeMA Journal, 75(2), 349-364.

[15] Akinyemi, L., Iyiola, O.S. (2020). Exact and approximate solutions of time-fractional models arising from physics via Shehu transform. Math. Meth. Appl. Sci., 1-23. https://doi.org/10.1002/mma.6484

[16] Mathanaranjan. T, Himalini. K. (2019) Analytical solutions of the time-fractional non-linear Schrodinger equation with zero and non zero trapping potential through the Sumudu Decomposition method. J Sci Univ Kelaniya, 12, 21-33.

[17] Mathanaranjan. T, Vijayakumar. D. (2021). Laplace Decomposition Method for TimeFractional Fornberg-Whitham Type Equations. Journal of Applied Mathematics and Physics, 9, 260-271.

[18] Akinyemi, L., Şenol, M., \& Iyiola, O. S. (2021) Exact solutions of the generalized multidimensional mathematical physics models via sub-equation method. Mathematics and Computers in Simulation, 182, 211-233. https://doi.org/10.1016/j.matcom.2020.10.017

[19] Wang, M., Li, X., \& Zhang, J. (2008). The $\left(G^{\prime} / G\right)$-expansion method and travelling wave solutions of nonlinear evolution equations in mathematical physics. Physics Letters A, 372(4), $417-423$. 
[20] Zayed, E. M. E., \& Gepreel, K. A. (2009). The $\left(G^{\prime} / G\right)$-expansion method for finding traveling wave solutions of nonlinear partial differential equations in mathematical physics. Journal of Mathematical Physics, 50(1), 013502.

[21] Zahran, E. H., \& Khater, M. M. (2014). The modified simple equation method and its applications for solving some nonlinear evolutions equations in mathematical physics. Jokull journal, 64(5), 297-312.

[22] Mathanaranjan, T. (2020). Solitary wave solutions of the Camassa-Holm-Nonlinear Schrödinger Equation. Results in Physics, 19, 103549.

[23] El-Wakil, S. A., \& Abdou, M. A. (2007). New exact travelling wave solutions using modified extended tanh-function method. Chaos, Solitons \& Fractals, 31(4), 840-852.

[24] Fan, E. (2000). Extended tanh-function method and its applications to nonlinear equations. Physics Letters A, 277(4-5), 212-218.

[25] Mathanaranjan. T. (2021). Soliton Solutions of Deformed Nonlinear Schrödinger Equations Using Ansatz Method. Int. J. Appl. Comput. Math. 7, 159.

[26] Sassaman. R, Biswas. A. (2010). Topological and non-topological solitons of the Klein-Gordon equations in 1+ 2 dimensions, Nonlinear Dyn. 61(1-2), 23-28.

[27] Vega-Guzman, J., Alshaery, A. A., Hilal, E. M., Bhrawy, A. H., Mahmood, M. F., Moraru, L., \& Biswas, A. (2014). Optical soliton perturbation in magneto-optic waveguides with spatiotemporal dispersion. Journal of Optoelectronics and Advanced Materials, 16(SeptemberOctober 2014), 1063-1070.

[28] Kara, A. H., Biswas, A., \& Belic, M. (2016). Conservation laws for optical solitons in birefringent fibers and magneto-optic waveguides. Optik, 127(24), 11662-11673.

[29] Dai, C. Q., Zhou, G. Q., Chen, R. P., Lai, X. J., \& Zheng, J. (2017). Vector multipole and vortex solitons in two-dimensional Kerr media. Nonlinear Dynamics, 88(4), 2629-2635.

[30] Asma, M., Biswas, A., Kara, A. H., Zayed, E. M., Guggilla, P., Khan, S., ... \& Belic, M. R. (2020). A pen-picture of solitons and conservation laws in magneto-optic waveguides having quadratic-cubic law of nonlinear refractive index. Optik, 223, 165330.

[31] Zayed, E. M., Alngar, M. E., El-Horbaty, M. M., Biswas, A., Guggilla, P., Ekici, M., ... \& Belic, M. R. (2020). Solitons in magneto-optic waveguides with parabolic law nonlinearity. Optik, 222, 165314.

[32] Zayed, E. M., Shohib, R. M., El-Horbaty, M. M., Biswas, A., Asma, M., Ekici, M., \& Belic, M. R. (2020). Solitons in magneto-optic waveguides with quadratic-cubic nonlinearity. Physics Letters A, 126456.

[33] Zayed, E. M., Alngar, M. E., Shohib, R. M., Biswas, A., Ekici, M., Alzahrani, A. K., \& Belic, M. R. (2020). Solitions in magneto-optic waveguides with anti-cubic nonlinearity. Optik, 222, 165313. 
[34] Biswas, A., Kara, A.H., Ekici, M. et al. (2021). Conservation Laws for Solitons in Magnetooptic Waveguides with Anti-cubic and Generalized Anti-cubic Nonlinearities. Regul. Chaot. Dyn. 26, 456-461. 